\title{
Analysis of muscle force in cycling and soccer athletes and regular gym participants: case-study
}

\begin{abstract}
Muscular strength is a primary biomechanical property that allows for human movement and can be defined as the ability of the muscular tissue to develop maximum strength from a muscle or muscle group at a given speed. However, when applied to the sports practice, muscle strength becomes more extensive, it is currently used differently for each sports modality since muscle tissue can generate force in different conditions of movement and physical demands. This study aimed to analyze the muscular strength of lower limbs of professional soccer and cycling athletes comparing with individuals gym participants. Sixty male subjects were selected for a convenience sample aged 18 to 40 years. Participants were divided into groups of 20 individuals from their sports modalities, being: SG-Soccer group, CG-Cycling and GG-Gym, matched by age, weight, height, and BMI. The participants of this research performed an isokinetic evaluation of the knee region. The data obtained by the isokinetic analysis of the torque peak (N.m) total work (J), agonist/antagonist ratio (\%) of the evaluated body segment were submitted to statistical analysis by the ANOVA test $(\mathrm{p} \leq 0.05)$ and Bonferroni test $(\mathrm{p} \leq 0.05)$. It was observed that at speeds of $60^{\circ} / \mathrm{s}$ and $180^{\circ} / \mathrm{s}$ there was a higher torque peak and total work for the SG and GG group with statistically significant values $(\mathrm{p} \leq 0.05)$, however, the agonist/antagonist ratio presented a higher relation for the CG group, the values were not significant $(\mathrm{p} \leq 0.05)$. In the multiple comparisons between the groups, it was observed that there was a difference in all groups evaluated $(\mathrm{p} \leq 0.05)$ at the speed of $60 \%$ (peak torque and total work) except for the group SG vs GG for flexor of the left knee. At the speed of $180^{\circ} / \mathrm{s}$ in torque peak was observed a significant difference ( $\mathrm{p} 0.05$ ), except for SG and GG (bilateral flexors and extensors). The total work in $180 \%$ s all groups there was difference ( $\mathrm{p} \leq 0.05$ ), except for CG vs GG (bilateral flexors) and the SG vs GG and GG vs CG (bilateral extensors). At speeds of $60 \%$ s and $180 \%$, the agonist/antagonist ratio was not significant for all groups evaluated. Thus, this study concludes that the SG group presented better values for all strength characteristics except for the agonist/antagonist ratio comparing from CG and GG group, followed for GG group.
\end{abstract}

Keywords: muscle strength, soccer, gym. cycling, isokinetic dynamometry
Volume 3 Issue 3 - 2018

\author{
Alexandre Badan Collucci, ${ }^{1,2}$ Eduardo \\ Guirado Campoi, ${ }^{3}$ Henrique Guirado \\ Campoi, ${ }^{3}$ Lucas Sartori Manoel,' Cássio \\ Mascarenhas Robert Pires,' Adilson \\ Aparecido Faccio, 'Veridiana Wanshi Arnoni,' \\ Bruno Ferreira, ${ }^{3}$ Carlos Alberto Giglio² \\ 'Study Center Collucci, Collucci's Clinical, Brazil \\ ${ }^{2}$ Department of Physical therapy, University UNAERP, Brazil \\ ${ }^{3}$ Department of Physical therapy, University Center UNIFAFIBE, \\ Brazil
}

Correspondence: Bruno Ferreira, Department of Physical therapy, University Center UNIFAFIBE, Bebedouro, Rua Wanda Bastos Santiago, 366, Ap I2, Bairro Jardim Botânico, I402 I-647, Ribeirão Preto, SP, Brazil,Tel +5516981934304, Email brunof22@me.com

Received: May 07, 2018 | Published: May 25, 2018

\section{Introduction}

Muscular strength is a primary biomechanical property that allows the humans to move and can be defined as the ability of muscle tissue to develop maximum strength from a muscle or muscle group at a given speed. ${ }^{1-3}$ In this way, in sports there is the use of muscular strength to measure and define the functional performance of an athlete is commonly employed, being classified as one of the main pillars of sports development. However, when applied to the sports practice, muscle strength becomes more extensive, it is currently used differently for each sports modality since muscle tissue can generate force in different conditions of movement and physical demands. ${ }^{1-3}$

The soccer performs depend directly on the muscular strength and anaerobic power of the neuromuscular system, especially the lower limb. During the time of a match, they perform kicks, jumps, attacks, and change in direction among others. Thus, the best teams invest more and more in the increase of their physical capacities, given the better performance of the players. ${ }^{4,5}$

During cycling activity, the interaction that occurs between the bicycle and the individual practitioner of this modality is complex, since it is necessary for the athlete to develop kinematics, strength and muscular activation to establish the best efficiency during cycling cycles, associated with an increase in its aerobic capacity. ${ }^{6}$
The gym training is the modality that develops the most throughout the world, being used to improve aspects related to strength. ${ }^{7}$ This modality uses the resistance training (washers, ballasts or plates of weights) existing in some apparatuses or implements to improve the physical capacity of the individual. It consists of resistance training, which can be performed with various loads, time, contraction range and controllable speed, presenting ease of adaptation to the physical condition of each, allowing even the training of sedentary people. ${ }^{8}$

Thus, the present study sought to compare the muscular strengths of lower limbs in professional soccer and cycling athletes and gym participants, identifying the peak torque, total work, agonist/antagonist ratio and muscular power of each type of sport. As a hypothesis of this study, it is expected that the soccer group presents values superior to the other groups in the variables peak of torque and total work, and the cyclist group presents higher power and muscular relation in comparison to the evaluated groups.

\section{Material and methods}

\section{Research characterization}

This observational cross-sectional case-study consists of pre-existing data collection in the Collucci Clinic Database and 
evaluation of muscular strength by isokinetic muscle dynamometry of cyclist and gym participants. It was approved by the Research Ethics Committee of the University of Ribeirão Preto (number CAE: 70170417.6.0000.5498).

\section{Characteristics of the population and sample}

Sixty male subjects that consisted of a convenience sample aged 18 to 40 years were selected, all physically active and without a clinical diagnosis of musculoskeletal diseases or postural alterations in the lower limbs, resident in the city of Ribeirão Preto-SP. Participants were divided into paired groups subject-to-subject from their sports modalities, being: SG - Soccer group: this group was constituted of 20 professional Soccer group players who have already performed isokinetic evaluation in the Collucci Clinic (data collection group, from the Collucci Clinic Database), CG-Cycling: 20 professional cycling athletes and GG-Gym: 20 regular gym participants with least three times a week activity, matched by age.

\section{Inclusion and exclusion criteria for the selection of individuals}

In the study were included individuals who were between the ages of 18 and 40, who did not present musculoskeletal conditions or postural changes in the lower limbs and should also be in the preseason, avoiding possible physical exhaustion that could influence the examination. The inclusion criteria applied was: SG- Soccer group participants needed to be professional players, previously hired by a soccer team; CG-Cycling group participants required being professional cyclists and employed and in the GG-Gym group participants needed to be practicing regular gym activity, performing it at least three times per week.

The exclusion criteria were: individuals who presented pain in the locomotor system or who used medication during the evaluation week, muscle injury and previously diagnosed chronic pathologies.

\section{Isokinetic evaluation}

In this study, the Biodex 4 Pro digital dynamometer (Biodex Medical System Inc., Shirley, NY, USA) was used to evaluate the quadriceps and hamstring muscle groups of the participants. Before the muscular evaluation, all of them underwent a previous exercise to avoid possible muscular injuries during ten minutes in an ergonomic bike RXV 1200 of the brand Total Health, with moderate intensity and light load according to the scale of Borg that could not exceed 10 points.

In the isokinetic dynamometry test, the subjects were positioned according to the recommendation of the standardization manual provided by the equipment manufacturer. The chest straps stabilized participants, with the " $\mathrm{x}$ " position, hip, and thigh of the assessed limb, to avoid possible incorrect movements during the test. After their fixation, the participants performed a familiarization session with the equipment at the same speeds of the test, so that potential biases in the design were reduced. ${ }^{9}$

In the evaluation protocol, the active range of joint motion of the knee was used in $90^{\circ}$ of flexion and full extension that could be maintained between the last 10 degrees of active movement of the knee. The rotation axis of the isokinetic dynamometer was aligned with the rotation axis of the knee joint tested (the dynamometer axis was aligned with the lateral condyle of the femur), a fact that avoided possible bias in the study. After this step, the limb was weighed in full extension, which served as normalization for the muscular strength reducing the interference of the action of gravity.

The tests were performed at the speed of 60 and $180^{\circ} / \mathrm{s}$ in the concentric mode, with five repetitions at $60 \%$ and twelve repetitions at $180^{\circ}$ s with 40 seconds of recovery. The tests always started with the dominant limb, and during the test, the volunteer was asked for the maximum voluntary contraction by a verbal stimulus.

\section{Evaluation of database}

The SG-Soccer group was obtained through the Clinic Collucci Database, in which the participants have already carried out a previous assessment with the purpose of verifying possible muscular changes in the years 2014 to 2015 . The data from the isokinetic system were retrieved since the isokinetic evaluations performed at the Collucci Clinic always follow the same pattern defined in this study.

For ethical reasons, the team that carried out the examinations was properly informed about the data usage; however, the name of the club, as well as the athlete, was kept in total secrecy.

\section{Isokinetic data analysis}

The values obtained by the isokinetic equipment were collected and used according to the following indicators: peak torque (N.m), total work $(J)$ and the agonist/antagonist ratio (\%) of the body segment, during the angular velocities of $60 \%$ s and $180 \%$ s.

\section{Statistical analysis}

The data obtained by isokinetic analysis of the peak torque, total work, the agonist/antagonist ratio of the evaluated body segment were submitted to statistical analysis using SPSS software version 21.0 (SPSS Inc., Chicago, IL, USA). The values were compared using the ANOVA test $(\mathrm{p}=0.05)$ to determine if there were changes in the biomechanical factors by the angular velocity used in the isokinetic and the Bonferroni test $(\mathrm{p}=0.05)$ to compare the groups and to evaluate the differences.

\section{Results}

\section{Sample distribution}

The population distribution of the sample in this study was paired subject-to-subject by the SG - Soccer group, CG-Cycling and GGGym groups. There was no statistically significant difference (Teste ANOVA; $\mathrm{p} \leq 0,05)$ in the comparison of the variable age $(\mathrm{SG}=26 \pm 0.89$ years, $C G=24 \pm 2.21$ years and $G G=25 \pm 0.90$ years with $p=0.81$ ), stature $(\mathrm{SG}=5.80 \pm 0.03$ feet, $\mathrm{CG}=5.70 \pm 0.03$ feet and $\mathrm{GG}=5.77 \pm 0.03$ feet with $\mathrm{p}=0.98)$ and $\mathrm{BMI}(\mathrm{SG}=24.16 \pm 0.37, \mathrm{CG}=23.09 \pm 0.45$ and $\mathrm{GG}=24.57 \pm 2.34$ with $\mathrm{p}=0.85$ ). However, the weight variable showed no significant difference in weight $(\mathrm{SG}=167.33 \pm 4.18 \mathrm{lbs}$, $\mathrm{CG}=154.43 \pm 2.82 \mathrm{lbs}$ and $\mathrm{GG}=169.53 \pm 3.61 \mathrm{lbs}$ with $\mathrm{p}=0.04)$.

\section{Isokinetic results comparing the groups evaluated in the speed of $60 \% \mathrm{~s}$}

In the analyzed condition of the peak torque by the speed of $60 \%$, it was observed a higher torque of the SG - Soccer group, followed by GG-Gym for all muscle groups evaluated in this study (Table 1) the results were statistically significant $(\mathrm{p} \leq 0.05)$.

Regarding the analysis of the total work, it was observed more work in the SG- Soccer group compared to GG- Gym and CG-Cycling for 
all muscles evaluated (Table 1) the results were statistically significant $(\mathrm{p} \leq 0.05)$.

In the analyzed condition of the relationship between agonist and antagonist, there was a higher ratio of the CG- Cycling group, followed by SG - Soccer group in both lower limbs (Table 1) and the results were not statistically significant $(\mathrm{p} \leq 0.05)$.

\section{Isokinetic results comparing the groups evaluated at the speed of e $180 \%$ s}

In the analyzed condition, peak torque in the speed of $180 \% \mathrm{~s}$ it was observed a higher power of all muscle groups evaluated in SGSoccer group, followed by the GG-Gym group (Table 2), values were statistically significant $(\mathrm{p} \leq 0.05)$.

In the analysis of the total work was verified a higher power of all muscle groups assessed from SG - Soccer, followed by GG - Gym group (Table 2 ), the values were statistically significant ( $\mathrm{p} \leq 0.05$ ).

In the analyzed condition of the relationship between agonist and antagonist, a higher ratio was found for the CG - Cycling group, followed by the GG - Gym group for both lower limbs (Table 2) the values found were not statistically significant $(\mathrm{p} \leq 0.05)$.

\section{Isokinetic results of the multiple comparisons of the groups evaluated at the speed of $60 \%$}

In the analysis of the peak torque of the right and left flexor and extensor muscles in the $60 \%$ it was observed a higher average difference between the groups for soccer and cycling when compared to the gym group for all the analyzed muscles (Table 3 ). These results are significant for all groups evaluated in all evaluated muscles except for the soccer-gym group for the right knee flexor muscles, right and left knee extensors $(\mathrm{p} \leq 0.05)$.

In the analysis of the total work of the right and left flexor and extensor muscles in the speed of $60 \%$ s there was a higher average difference between the groups for soccer and cycling when compared to the gym group, followed by the gym and cycling group in all muscles analyzed (Table 4). These results are significant for all groups evaluated except for the soccer group vs gym for all evaluated muscles $(\mathrm{p} \leq 0.05)$.

In the analysis of the agonist/antagonist ratio of the lower limbs in the speed of $60^{\circ} / \mathrm{s}$, there was a more substantial average difference for the cycling and gym group when compared to the soccer group in both lower limbs (Table 5). These results were not statistically significant $(\mathrm{p} \leq 0.05)$.

\section{Isokinetic results of the multiple comparisons of the groups evaluated at the speed of $180 \%$}

In the analysis of the peak torque of the right and left flexor and extensor muscles in the speed of $180^{\circ} / \mathrm{s}$, there was a higher average difference between the groups for soccer and cycling when compared to the gym group for all muscles analyzed (Table 6). These results are significant for all groups evaluated in all evaluated muscles except for the soccer-gym group for the right knee flexor muscles, right and left knee extensors $(\mathrm{p} \leq 0.05)$.

In the analysis of the total work of the right and left flexor and extensor muscles in the speed of $180^{\circ}$ /s there was a higher average difference between the groups for soccer and cycling when compared to the gym group (Table 7). These results are significant for all groups evaluated except for the gym vs cycling group for all assessed muscles $(\mathrm{p} \leq 0.05)$.

In the analysis of the agonist/antagonist ratio of the lower limbs in the speed of $180^{\circ} / \mathrm{s}$, there was a more substantial average difference for the cycling and gym group when compared to the soccer group in both lower limbs (Table 8). These results were not statistically significant $(\mathrm{p} \leq 0.05)$.

Table I Results of the average and standard error of the isokinetic evaluation in the speed of $60^{\circ} / \mathrm{s}$ for the group SG-Soccer group, CG-Cycling and GG-Gym for the analyzed conditions peak torque (N.m) of the right and left flexors and extensors, total work (J) of the right and left flexors and extensors, and the relationship between right and left agonist and antagonist (\%) (ANOVA test, $\mathrm{p} \leq 0.05$ )

\begin{tabular}{|c|c|c|c|c|}
\hline Condition analyzed & Groups & P value & Average & Standard error \\
\hline \multirow{3}{*}{ Right flexors torque peak } & SG-Soccer & & 142.68 & \pm 6.27 \\
\hline & CG-Cycling & $0.00 * *$ & 100.63 & \pm 5.14 \\
\hline & GG-Gym & & 129.36 & \pm 5.69 \\
\hline \multirow{3}{*}{ Left flexors torque peak } & SG-Soccer & & 145.83 & \pm 5.27 \\
\hline & CG-Cycling & $0.00^{* *}$ & 98.88 & \pm 4.63 \\
\hline & GG-Gym & & 125.42 & \pm 4.84 \\
\hline \multirow{3}{*}{ Right extenders torque peak } & SG-Soccer & & 265.15 & \pm 10.43 \\
\hline & CG-Cycling & $0.00 * *$ & 184.85 & \pm 8.87 \\
\hline & GG-Gym & & 242.25 & \pm 10.53 \\
\hline \multirow{3}{*}{ Left extenders torque peak } & SG-Soccer & & 270.06 & \pm 9.84 \\
\hline & CG-Cycling & $0.00 * *$ & 186.86 & \pm 9.77 \\
\hline & GG-Gym & & 237.89 & \pm 9.53 \\
\hline \multirow{3}{*}{ Total work right flexors } & SG-Soccer & & 691.03 & \pm 40.95 \\
\hline & CG-Cycling & $0.00^{* *}$ & 500.33 & \pm 23.40 \\
\hline & GG-Gym & & 637.45 & \pm 40.35 \\
\hline
\end{tabular}


Table Continued

\begin{tabular}{lllll}
\hline Condition analyzed & Groups & P value & Average & Standard error \\
\hline \multirow{2}{*}{ Total work left flexors } & SG-Soccer & & 705.08 & \pm 39.10 \\
& CG-Cycling & $0.00^{* *}$ & 479.63 & \pm 25.40 \\
& GG-Gym & & 628.07 & \pm 31.46 \\
\hline \multirow{2}{*}{ Total work right extenders } & SG-Soccer & & 1021.64 & \pm 62.87 \\
& CG-Cycling & $0.00^{* *}$ & 776.97 & \pm 40.16 \\
& GG-Gym & & 1008.70 & \pm 60.00 \\
Total work left extenders & SG-Soccer & & 1068.34 & \pm 59.25 \\
& CG-Cycling & $0.00^{* *}$ & 786.83 & \pm 45.68 \\
& GG-Gym & & 1041.54 & \pm 33.28 \\
& SG-Soccer & & 54.28 & \pm 1.75 \\
Right agonist/antagonist & CG-Cycling & $0.46^{\text {ns }}$ & 57.08 & \pm 2.55 \\
ratio & GG-Gym & & 53.52 & \pm 1.95 \\
& SG-Soccer & 54.33 & \pm 1.87 \\
& CG-Cycling & $0.49^{\text {ns }}$ & 55.75 & \pm 2.16 \\
& GG-Gym & 52.43 & \pm 1.81 \\
\hline & & &
\end{tabular}

$* *$-Significant values $(\mathrm{p} \leq 0.0 \mathrm{I})$

ns-Not significant $(p \leq 0.05)$

Table 2 Results of the average and standard error of the isokinetic evaluation in the speed of $180^{\circ}$ s for the SG- Soccer group, CG-Cycling and GG-Gym for the analyzed conditions of peak torque (N.m) of the right and left flexors and extensors, total work (J) of the right and left flexors and extensors, and the relationship between right and left agonist and antagonist (\%) (ANOVA test, $\mathrm{p} \leq 0.05$ )

\begin{tabular}{|c|c|c|c|c|}
\hline Condition Analyzed & Groups & P Value & Average & Standard Error \\
\hline \multirow{3}{*}{ Right flexors torque peak } & SG-Soccer & \multirow{3}{*}{$0.00 * *$} & 114.51 & \pm 4.81 \\
\hline & CG-Cycling & & 87.31 & \pm 4.11 \\
\hline & GG-Gym & & 105.21 & \pm 5.16 \\
\hline \multirow{3}{*}{ Left flexors torque peak } & SG-Soccer & \multirow{3}{*}{$0.00 * *$} & 116.00 & \pm 4.46 \\
\hline & CG-Cycling & & 86.41 & \pm 4.62 \\
\hline & GG-Gym & & 102.98 & \pm 5.13 \\
\hline \multirow{3}{*}{ Right extenders torque peak } & SG-Soccer & \multirow{3}{*}{$0.00 * *$} & 181.40 & \pm 7.46 \\
\hline & CG-Cycling & & 127.24 & \pm 4.92 \\
\hline & GG-Gym & & 176.45 & \pm 9.02 \\
\hline \multirow{3}{*}{ Left extenders torque peak } & SG-Soccer & \multirow{3}{*}{$0.00 * *$} & 186.12 & \pm 6.19 \\
\hline & CG-Cycling & & 126.26 & \pm 5.93 \\
\hline & GG-Gym & & 171.26 & \pm 6.41 \\
\hline \multirow{3}{*}{ Total work right flexors } & SG-Soccer & \multirow{3}{*}{$0.00 * *$} & 1478.70 & \pm 124.84 \\
\hline & CG-Cycling & & 899.94 & \pm 48.01 \\
\hline & GG-Gym & & 1021.63 & \pm 72.17 \\
\hline \multirow{3}{*}{ Total work left flexors } & SG-Soccer & \multirow{3}{*}{$0.00 * *$} & 1521.07 & \pm 134.70 \\
\hline & CG-Cycling & & 846.61 & \pm 44.94 \\
\hline & GG-Gym & & 991.53 & \pm 62.01 \\
\hline \multirow{3}{*}{ Total work right extenders } & SG-Soccer & \multirow{3}{*}{$0.00 * *$} & 2135.74 & \pm 169.91 \\
\hline & CG- Cycling & & 1451.67 & \pm 67.28 \\
\hline & GG-Gym & & 1768.71 & \pm 120.35 \\
\hline
\end{tabular}


Table Continued

\begin{tabular}{lllll}
\hline Condition Analyzed & Groups & P Value & Average & Standard Error \\
\hline \multirow{3}{*}{ Total work left extenders } & SG-Soccer & & 2090.03 & \pm 201.29 \\
& CG-Cycling & $0.00^{* *}$ & 1432.75 & \pm 66.71 \\
& GG-Gym & & 1741.52 & \pm 107.83 \\
& SG-Soccer & & 63.73 & \pm 2.47 \\
Right agonist/antagonist ratio & CG-Cycling & $0.77^{\text {ns }}$ & 66.68 & \pm 1.97 \\
& GG-Gym & & 59.99 & \pm 1.61 \\
& SG-Soccer & & 62.41 & \pm 1.72 \\
Left agonist/antagonist ratio & CG-Cycling & $0.70^{\text {ns }}$ & 66.41 & \pm 1.79 \\
& GG-Gym & & 60.25 & \pm 2.09
\end{tabular}

**-Significant values $(\mathrm{p} \leq 0.01)$

ns-Not significant $(p \leq 0.05)$

Table 3 Results of the intergroup analysis of the average differences and standard error of the isokinetic evaluation in the speed of $60^{\circ} / \mathrm{s}$ comparing the SGSoccer group, CG-Cycling and GG-Gym groups, for the analyzed peak torque conditions (N.m) of the right and left flexors and extensors (Bonferroni test, $\mathrm{p} \leq 0.05)$

\begin{tabular}{|c|c|c|c|c|}
\hline Muscles & Compared groups & P value & Average difference & Standard error \\
\hline \multirow{6}{*}{ Right knee flexors } & Soccer vs Cycling & $0.00 * *$ & 42.05 & 8.09 \\
\hline & Soccer vs Gym & $0.31^{\mathrm{ns}}$ & 13.32 & \pm 8.09 \\
\hline & Cycling vs Soccer & $0.00 * *$ & -42.05 & \pm 8.09 \\
\hline & Cycling vs Gym & $0.00 * *$ & -28.72 & \pm 8.09 \\
\hline & Gym vs Soccer & $0.31^{\mathrm{ns}}$ & -13.32 & \pm 8.09 \\
\hline & Gym vs Cycling & $0.00 * *$ & 28.72 & \pm 8.09 \\
\hline \multirow{6}{*}{ Left knee flexors } & Soccer vs Cycling & $0.00 * *$ & 46.95 & \pm 6.96 \\
\hline & Soccer vs Gym & $0.01 * *$ & 20.41 & \pm 6.96 \\
\hline & Cycling vs Soccer & $0.00 * *$ & -46.95 & \pm 6.96 \\
\hline & Cycling vs Gym & $0.00 * *$ & -26.54 & \pm 6.96 \\
\hline & Gym vs Soccer & $0.01 * *$ & -20.41 & \pm 6.96 \\
\hline & Gym vs Cycling & $0.00 * *$ & 26.54 & \pm 6.96 \\
\hline \multirow{6}{*}{ Right knee extenders } & Soccer vs Cycling & $0.00 * *$ & 80.30 & \pm 14.11 \\
\hline & Soccer vs Gym & $0.33^{\mathrm{ns}}$ & 22.90 & \pm 14.11 \\
\hline & Cycling vs Soccer & $0.00 * *$ & -80.30 & \pm 14.11 \\
\hline & Cycling vs Gym & $0.00 * *$ & -57.40 & \pm 14.11 \\
\hline & Gym vs Soccer & $0.33^{\mathrm{ns}}$ & -22.90 & \pm 14.11 \\
\hline & Gym vs Cycling & $0.00 * *$ & 57.40 & \pm 14.11 \\
\hline \multirow{6}{*}{ Left knee extenders } & Soccer vs Cycling & $0.00 * *$ & 83.20 & \pm 2.98 \\
\hline & Soccer vs Gym & $0.06^{\mathrm{ns}}$ & 32.17 & \pm 2.98 \\
\hline & Cycling vs Soccer & $0.00 * *$ & -83.20 & \pm 2.98 \\
\hline & Cycling vs Gym & $0.00 * *$ & -51.02 & \pm 2.98 \\
\hline & Gym vs Soccer & $0.68^{\text {ns }}$ & 32.17 & \pm 2.98 \\
\hline & Gym vs Cycling & $0.00 * *$ & 51.02 & \pm 2.98 \\
\hline
\end{tabular}

**-Significant value $(p \leq 0.01)$

ns-Not significant $(p \leq 0.05)$ 
Table 4 Results of the intergroup analysis of the average differences and standard error of the isokinetic evaluation in the speed of $60 \%$ s comparing the groups SG-Soccer group, CG-Cycling and GG-Gym, for the analyzed conditions of total work (J) of the right and left flexors and extensors (Bonferroni test, $\mathrm{p} \leq 0.05$ )

\begin{tabular}{|c|c|c|c|c|}
\hline Muscles & Compared groups & P value & Average difference & Standard error \\
\hline \multirow{6}{*}{ Right knee flexors } & Soccer vs Cycling & $0.00 * *$ & 190.69 & 50.69 \\
\hline & Soccer vs Gym & $0.88^{\text {ns }}$ & 53.58 & \pm 50.69 \\
\hline & Cycling vs Soccer & $0.00 * *$ & -190.69 & \pm 50.69 \\
\hline & Cycling vs Gym & $0.02 *$ & -137.11 & \pm 50.69 \\
\hline & Gym vs Soccer & $0.88^{\mathrm{ns}}$ & -53.58 & \pm 50.69 \\
\hline & Gym vs Cycling & $0.02 *$ & 137.11 & \pm 50.69 \\
\hline \multirow{6}{*}{ Left knee flexors } & Soccer vs Cycling & $0.00 * *$ & 225.45 & 45.93 \\
\hline & Soccer vs Gym & $0.29^{\mathrm{ns}}$ & 77.01 & \pm 45.93 \\
\hline & Cycling vs Soccer & $0.00 * *$ & -225.45 & \pm 45.93 \\
\hline & Cycling vs Gym & $0.00 * *$ & -148.44 & \pm 45.93 \\
\hline & Gym vs Soccer & $0.29^{\mathrm{ns}}$ & -77.01 & \pm 45.93 \\
\hline & Gym vs Cycling & $0.00 * *$ & 148.44 & \pm 45.93 \\
\hline \multirow{6}{*}{ Right knee extenders } & Soccer vs Cycling & $0.00 * *$ & 244.67 & 78.17 \\
\hline & Soccer vs Gym & $1.00^{\mathrm{ns}}$ & 12.94 & \pm 78.17 \\
\hline & Cycling vs Soccer & $0.00 * *$ & -244.64 & \pm 78.17 \\
\hline & Cycling vs Gym & $0.01 * *$ & -231.73 & \pm 78.17 \\
\hline & Gym vs Soccer & $1.00^{\mathrm{ns}}$ & -12.94 & \pm 78.17 \\
\hline & Gym vs Cycling & $0.01 * *$ & 231.73 & \pm 78.17 \\
\hline \multirow{6}{*}{ Left knee extenders } & Soccer vs Cycling & $0.00 * *$ & 281.51 & 72.38 \\
\hline & Soccer vs Gym & $1.00^{\mathrm{ns}}$ & 26.80 & \pm 71.44 \\
\hline & Cycling vs Soccer & $0.00 * *$ & -281.51 & \pm 72.38 \\
\hline & Cycling vs Gym & $0.00 * *$ & -254.71 & \pm 72.38 \\
\hline & Gym vs Soccer & $1.00^{\mathrm{ns}}$ & -26.80 & \pm 71.44 \\
\hline & Gym vs Cycling & $0.00 * *$ & 254.71 & \pm 72.38 \\
\hline
\end{tabular}

**-Significant value $(\mathrm{p} \leq 0.0 \mathrm{I})$

Gym vs Cycling

$0.00 * * \quad 254.71$

*_Significant value $(p \leq 0.05)$

ns-Not significant $(p \leq 0.05)$

Table 5 Results of the intergroup analysis of the average differences and standard error of the isokinetic evaluation in the speed of $60^{\circ} / \mathrm{s}$ comparing the groups SG-Soccer group, CG-Cycling and GG-Gym, for the analyzed conditions of agonist/antagonist ratio (\%) of the right and left lower limb (Bonferroni test, $\mathrm{p} \leq 0.05)$

\begin{tabular}{|c|c|c|c|c|}
\hline Relationship & Compared Groups & P Value & Average Difference & Standard Error \\
\hline \multirow{6}{*}{ Agonist/antagonist ratio of right lower limb } & Soccer vs Cycling & $1.00^{\mathrm{ns}}$ & -2.80 & \pm 2.98 \\
\hline & Soccer vs Gum & $1.00^{\mathrm{ns}}$ & 0.76 & \pm 2.98 \\
\hline & Cycling vs Soccer & $1.00^{\mathrm{ns}}$ & 2.80 & \pm 2.98 \\
\hline & Cycling vs Gym & $0.71^{\mathrm{ns}}$ & 3.56 & \pm 2.98 \\
\hline & Gym vs Soccer & $1.00^{\mathrm{ns}}$ & -0.76 & \pm 2.98 \\
\hline & Gym vs Cycling & $0.71^{\mathrm{ns}}$ & -3.56 & \pm 2.98 \\
\hline \multirow{6}{*}{ Agonist/antagonist ratio of the left lower limb } & Soccer vs Cycling & $1.00^{\mathrm{ns}}$ & -1.42 & \pm 2.76 \\
\hline & Soccer vs Gum & $1.00^{\mathrm{ns}}$ & 1.89 & \pm 2.76 \\
\hline & Cycling vs Soccer & $1.00^{\mathrm{ns}}$ & 1.42 & \pm 2.76 \\
\hline & Cycling vs Gym & $0.70^{\mathrm{ns}}$ & 3.31 & \pm 2.76 \\
\hline & Gym vs Soccer & $1.00^{\mathrm{ns}}$ & -1.89 & \pm 2.76 \\
\hline & Gym vs Cycling & $0.70^{\mathrm{ns}}$ & -3.31 & \pm 2.76 \\
\hline
\end{tabular}

ns-Not significant $(p \leq 0.05)$

Table 6 Results of the intergroup analysis of the average differences and standard error of the isokinetic evaluation in the speed of $180^{\circ} / \mathrm{s}$ comparing the SGSoccer group, CG-Cycling and GG-Gym groups, for the analyzed peak torque conditions (N.m) of the right and left flexors and extensors (Bonferroni test, $\mathrm{p} \leq 0.05)$

\begin{tabular}{lllll}
\hline Muscles & Compared groups & P value & Average difference & Standard error \\
\hline \multirow{4}{*}{ Right knee flexors } & Soccer vs Cycling & $0.00^{* *}$ & 27.20 & \pm 6.67 \\
& Soccer vs Gym & $0.50^{\text {ns }}$ & 9.30 & \pm 6.67 \\
& Cycling vs Soccer & $0.00^{* *}$ & -27.20 & \pm 6.67 \\
& Cycling vs Gym & $0.02^{*}$ & -17.90 & \pm 6.67 \\
& Gym vs Soccer & $0.50^{\text {ns }}$ & -9.30 & \pm 6.67 \\
& Gym vs Cycling & $0.02^{*}$ & 17.90 & \pm 6.67
\end{tabular}


Table Continued

\begin{tabular}{lllll}
\hline Muscles & Compared groups & P value & Average difference & Standard error \\
\hline \multirow{4}{*}{ Left knee flexors } & Soccer vs Cycling & $0.00^{* *}$ & 29.59 & \pm 6.71 \\
& Soccer vs Gym & $0.17^{\text {ns }}$ & 13.02 & \pm 6.71 \\
& Cycling vs Soccer & $0.00^{* *}$ & -29.59 & \pm 6.71 \\
& Cycling vs Gym & $0.05^{*}$ & -16.57 & \pm 6.71 \\
& Gym vs Soccer & $0.17^{\text {ns }}$ & -13.20 & \pm 6.71 \\
& Gym vs Cycling & $0.05^{*}$ & 16.57 & \pm 6.71 \\
Right knee extenders & Soccer vs Cycling & $0.00^{* *}$ & 54.16 & 10.37 \\
& Soccer vs Gym & $1.00^{\text {ns }}$ & 4.95 & \pm 10.37 \\
& Cycling vs Soccer & $0.00^{* *}$ & -54.16 & \pm 10.37 \\
& Cycling vs Gym & $0.00^{* *}$ & -49.21 & \pm 10.37 \\
& Gym vs Soccer & $1.00^{\text {ns }}$ & -4.95 & \pm 10.37 \\
& Gym vs Cycling & $0.00^{* *}$ & 49.21 & \pm 10.37 \\
& Soccer vs Cycling & $0.00^{* *}$ & 59.85 & 8.87 \\
& Soccer vs Gym & $0.28^{\text {ns }}$ & 14.85 & \pm 8.87 \\
Left knee extenders & Cycling vs Soccer & $0.00^{* *}$ & -59.85 & \pm 8.87 \\
& Cycling vs Gym & $0.00^{* *}$ & -45.00 & \pm 8.87 \\
& Gym vs Soccer & $0.28^{\text {ns }}$ & -14.85 & \pm 8.87 \\
& Gym vs Cycling & $0.00^{* *}$ & 45.00 & \pm 8.87 \\
\hline
\end{tabular}

**-Significant value $(\mathrm{p} \leq 0.0 \mathrm{I})$

*-Significant value $(\mathrm{p} \leq 0.05)$

ns-Not significant $(\mathrm{p} \leq 0.05)$

Table 7 Results of the intergroup analysis with average differences and standard error of the isokinetic evaluation in the speed of I80\% comparing SGSoccer group, CG-Cycling and GG-Gym, for the analyzed conditions of total work (J) of the right and left flexors and extensors (Bonferroni test, $\mathrm{p} \leq 0.05$ )

\begin{tabular}{|c|c|c|c|c|}
\hline Muscles & Compared groups & P value & Average difference & Standard error \\
\hline \multirow{6}{*}{ Right knee flexors } & Soccer vs Cycling & $0.00 * *$ & 578.76 & 124.09 \\
\hline & Soccer vs Gym & $0.00 * *$ & 457.06 & \pm 124.09 \\
\hline & Cycling vs Soccer & $0.00 * *$ & -578.76 & \pm 124.09 \\
\hline & Cycling vs Gym & $0.99^{\text {ns }}$ & -121.69 & \pm 124.09 \\
\hline & Gym vs Soccer & $0.00 * *$ & -457.06 & \pm 124.09 \\
\hline & Gym vs Cycling & $0.99^{\text {ns }}$ & 121.69 & \pm 124.09 \\
\hline \multirow{6}{*}{ Left knee flexors } & Soccer vs Cycling & $0.00 * *$ & 674.46 & 126.52 \\
\hline & Soccer vs Gym & $0.00 * *$ & 529.54 & \pm 126.52 \\
\hline & Cycling vs Soccer & $0.00 * *$ & -674.46 & \pm 126.52 \\
\hline & Cycling vs Gym & $0.77^{\mathrm{ns}}$ & -144.92 & \pm 126.52 \\
\hline & Gym vs Soccer & $0.00 * *$ & -529.54 & \pm 126.52 \\
\hline & Gym vs Cycling & $0.77^{\mathrm{ns}}$ & 144.92 & \pm 126.52 \\
\hline \multirow{6}{*}{ Right knee extenders } & Soccer vs Cycling & $0.00 * *$ & 683.76 & 178.66 \\
\hline & Soccer vs Gym & $0.13^{\mathrm{ns}}$ & 366.72 & \pm 178.66 \\
\hline & Cycling vs Soccer & $0.00 * *$ & 683.76 & \pm 178.66 \\
\hline & Cycling vs Gym & $0.24^{\mathrm{ns}}$ & -317.04 & \pm 178.66 \\
\hline & Gym vs Soccer & $0.13^{\mathrm{ns}}$ & -366.72 & \pm 178.66 \\
\hline & Gym vs Cycling & $0.24^{\mathrm{ns}}$ & 317.04 & \pm 178.66 \\
\hline \multirow{6}{*}{ Left knee extenders } & Soccer vs Cycling & $0.00 * *$ & 657.28 & 194.25 \\
\hline & Soccer vs Gym & $0.23^{\mathrm{ns}}$ & 348.51 & \pm 194.25 \\
\hline & Cycling vs Soccer & $0.00 * *$ & -657.28 & \pm 194.25 \\
\hline & Cycling vs Gym & $0.35^{\mathrm{ns}}$ & -308.77 & \pm 194.25 \\
\hline & Gym vs Soccer & $0.23^{\mathrm{ns}}$ & -347.51 & \pm 194.25 \\
\hline & Gym vs Cycling & $0.35^{\mathrm{ns}}$ & 308.77 & \pm 194.25 \\
\hline
\end{tabular}

**-Significant value $(\mathrm{p} \leq 0.01)$

ns-Not significant $(\mathrm{p} \leq 0.05)$

Citation: Collucci AB, Campoi EG, Campoi HG, et al.Analysis of muscle force in cycling and soccer athletes and regular gym participants: case-study. Int Phys Med Rehab J. 2018;3(3):224-232. DOI: 10.15406/ipmrj.2018.03.00108 
Table 8 Results of the intergroup analysis with average differences and Soccer group, CG-Cycling and GG-Gym, for the analyzed conditions of standard error of the isokinetic evaluation in the speed of $180^{\circ} / \mathrm{s}$ comparing SG- agonist / antagonist ratio (\%) of the right and left lower limb (Bonferroni test, $\mathrm{p} \leq 0.05$ ).

\begin{tabular}{|c|c|c|c|c|}
\hline Relationship & Compared groups & Pvalue & Average difference & Standard error \\
\hline \multirow{6}{*}{ Agonist/antagonist ratio of right lower limb } & Soccer vs Cycling & $0.94^{\mathrm{ns}}$ & -2.95 & \pm 2.90 \\
\hline & Soccer vs Gym & $0.60^{\mathrm{ns}}$ & 3.74 & \pm 2.90 \\
\hline & Cycling vs Soccer & $0.94^{\mathrm{ns}}$ & 2.95 & \pm 2.90 \\
\hline & Cycling vs Gym & $0.07^{\mathrm{ns}}$ & 6.69 & \pm 2.90 \\
\hline & Gym vs Soccer & $0.60^{\text {ns }}$ & -3.74 & \pm 2.90 \\
\hline & Gym vs Cycling & $0.07^{\mathrm{ns}}$ & -6.69 & \pm 2.90 \\
\hline Relationship & Compared groups & P value & Average difference & Standard error \\
\hline \multirow{6}{*}{ Agonist/antagonist ratio of the left lower limb } & Soccer vs Cycling & $0.41^{\mathrm{ns}}$ & -4.00 & \pm 2.65 \\
\hline & Soccer vs Gym & $1.00^{\mathrm{ns}}$ & 2.16 & \pm 2.65 \\
\hline & Cycling vs Soccer & $0.41^{\mathrm{ns}}$ & 4.00 & \pm 2.65 \\
\hline & Cycling vs Gym & $0.07^{\mathrm{ns}}$ & 6.16 & \pm 2.65 \\
\hline & Gym vs Soccer & $1.00^{\text {ns }}$ & -2.16 & \pm 2.65 \\
\hline & Gym vs Cycling & $0.07^{\mathrm{ns}}$ & -6.16 & \pm 2.65 \\
\hline
\end{tabular}

**-Significant value $(p \leq 0.01)$

ns-Not significant $(p \leq 0.05)$

\section{Discussion}

Muscular strength is a biomechanical property that has a influence on the performance of the athletic movement, and when compared to the performance of the athlete it becomes even more relevant since the improvement of this force allows higher performance in the sport. ${ }^{10}$

In this way, this study aimed to compare the muscular strength of different types of physical activity so that it is possible to observe the difference existing in each group, which will contribute to better rehabilitate each individual within their sports modalities by working the specificities of each modality during the rehabilitation phase.

In present study, the groups evaluated were SG-Soccer group, CG-Cycling and GG-Gym. They were paired subject-to-subject by age, weight, height and BMI, demonstrating that the individuals participating in each group are the same, except for the variable weight of the cyclist group, these findings corroborate with Mujika et al. ${ }^{11}$ who report that there is a high energy production associated with sports spending in cyclists, thus reducing the body mass rate in their bodies, favoring the reduction of the body weight of these athletes. This fact may be justifiable, since according to Borszcz et al. ${ }^{12}$ describe that the cyclists present in their practice a resistance performance regarding physiological characteristics and that it is related to the development of maximum aerobic power before the training, thus remitting that the practice of cycling has a profile of higher aerobic requirement.

In the SG - Soccer group movements such as change of direction, sprints, acceleration, and deceleration, as well as jumps, which favors, according to Tonnessen, et al. ${ }^{13}$ and Stolen et al. ${ }^{14}$ a predominance of the aerobic system, but with important participation of the anaerobic system due to large amount of explosive movements during the game. What justifies the results found of a difference of body weight only in the CG-Cycling, since the practice of gym also uses of the same corporal capacity that the soccer, because according to Reis et al $^{15}$ where they investigated the energy expenditures in different intensities of the most used resistance exercises, they could conclude that the high intensity of the workouts in $80 \%$ of $1-\mathrm{RM}$, that is to say, a force work, obtained a predominance of energy release via anaerobic for lower limbs, which can be justified by Scott et al. ${ }^{16}$ that muscles classified as skeletal have a restricted reserve of ATP and creatine phosphate.

In the evaluation of muscular strength, it was observed that in the assessment of the peak of muscular torque comparing the groups evaluated in the speed of $60 \% \mathrm{~s}$ and $180 \% \mathrm{~s}$ there was higher torque in the SG-Soccer group followed by the GG-Gym group for all muscle groups evaluated in the study, corroborating the study of Paul et al. ${ }^{17}$ who observed the predominance of muscular strength in soccer athletes and reported that the athletic movements performed during the game use essential characteristics of power and muscular strength to develop jumps, sprints, kicks among others that are movements carried out on a large scale and frequently by the athletes of this modality. Thus, our findings are justified, since the position in the field in which each player performs his function generates higher relative values of muscular strength in the isokinetic apparatus which may have contributed to observe large values of the SG-Soccer group in the present study. ${ }^{18}$

In the analysis of the total work from the comparison between the groups evaluated in the speed of $60 \% \mathrm{~s}$ and $180 \% \mathrm{~s}$ it was verified that the CG-Cycling group presented the lowest strength for total work in comparison to the other groups, which is justifiable since the hip position interferes in the performance of muscular strength and these individuals, they play their seated sports movements which harm knee muscle strength. We believe that these findings occurred in our study because there was the action of the muscle-length relationship theory observing that muscles with smaller length tend to generate less force. ${ }^{19}$

For the SG-Soccer group, it presented higher total work in comparison to the GG-Gym and CG-Cycling group for all evaluated muscles, and this total work can be understood as the ratio of the 
force played by the muscles against a resistance throughout the arch of motion, considering in a high intensity. However, no articles were found that evidenced the total work performed by the groups evaluated in this research for a possible comparison..$^{20,21}$

In the evaluation of the relationship between agonist and antagonist muscles, there was no significant statistical change between CGCycling followed by SG-Soccer group, since the evaluation of muscle strength compares the proportion between flexors and thigh extensors and in these athletes is it was possible to observe that there was no significant change in muscle strength between the groups, since they did not present muscle injury and/or time of inactivity of the sports practice. However, it was not possible to find studies in the literature that explicitly described the interaction of the agonist/antagonist ratio as a clinical criterion, being that this data is fundamental during our clinical practice, thus allowing real data of the balance of force exists in patients who should be work on improving their recovery.

This study had limitations in its development, because it was not possible to find studies comparing the three modalities used and comparing the biomechanical capacities of muscular strength, which made it difficult to develop the discussion, it was also possible to observe that the other muscular strength data are not very explored in the current literature and have a substantial impact on the clinical applicability of the patient, and may even be criteria for high physiotherapy.

In this way, the present study can show that during the isokinetic evaluation by the speed of $60 \%$ and $180 \%$ s the SG-Soccer group presented higher values of peak torque and total muscular work of all muscle groups evaluated when compared to other activities. In the values found in the agonist and antagonist ratio in the speed of $60^{\circ} / \mathrm{s}$ and $180^{\circ}$ s there was a higher average difference for the cycling and gym group when compared to the soccer group, but they were not statistically significant.

This study has high clinical relevance for identifying the degree of muscular strength present in the different physical activities, allowing a more significant understanding of prevention and performance work in sports, improving the physiotherapeutic actuation through evidence-based physiotherapy.

It is concluded that the practice of soccer develops more torque pike, total work comparing from CG-Cycling and GG-Gym group, followed for GG group, except for agonist/antagonist ratio who presented better value for the CG-Cycling group.

\section{Acknowledgement}

None.

\section{Conflict of interest}

The Authors declare no conflict of interests.

\section{References}

1. Sapega AA, Drillings G. The definition and assessment of muscular power. J Orthop Sports Phys Ther. 1983;5(1):7-9.

2. Enoka RM. Muscle Strengh and Its Development - New Perspectives. Sports Medicine. 1988;6(3):146-168.

3. Perez CM, Teixeira FS, Gonzalo RF, et al. Effects of high-speed power training on muscle strength and power in patients with multiple sclerosis. J Rehabil Res Dev. 2016;53(3):359-368.
4. Lehance C, Binet J, Bury T, Croisier JL (2009) Muscular strength, functional performances and injury risk in professional and junior elite soccer players. Scandinavian Scand J Med Sci Sports. 19(2):243-251.

5. Negra Y, Chaabene H, Sammoud S, et al. Effects of Plyometric Training on Components of Physical Fitness in Prepuberal Male Soccer Athletes: The Role of Surface Instability. J Strength Cond Res. 2017;31(12):32953304.

6. Sanders D, Heiiboer M, Hesselink MKC, et al. Analysing a cycling grand tour: Can we monitor fatigue with intensity or load ratios?. $J$ Sports Sci. 2018;36(12):1385-1391.

7. Gore D, Kothari A. Social determinants of health in Canada: Are healthy living initiatives there yet? A policy analysis. Int $J$ Equity Health. 2012;11:41

8. Rodríguez RD. Yáñez GJM, Torres TJ, et al. Effort Index as a Novel Variable for Monitoring the Level of Effort During Resistance Exercises. J Strength Cond Res. 2018.

9. Cvjetkovic DD, Bijeljac, S, Palija S, et al Isokinetic Testing in Evaluation Rehabilitation Outcome After ACL Reconstruction. Med Arch. 2015;69(1):21-23.

10. Suchomel TJ, Nimphiius S, Stone MH. The importance of muscular strength in athletic performance. Sports Med. 2016;46(10): 1413-1449.

11. Mujika I, Ronnestad BR, Martin DT. Effects of Increased Muscle Strength and Muscle Mass on Endurance-Cycling Performance. Int J Sports Physiol Perform. 2016;11(3):283-289.

12. Borszcz FK, Tramontin AF, Souza KM, et al. Physiological Correlations With Short, Medium, and Long Cycling Time-Trial Performance. Res $Q$ Exerc Sport. 2018; 89(1):120-125.

13. Tonnessen E, Shalfawi SAI, Haugen T, et al. The effect of 40-m repeated sprint training on maximum sprinting speed, repeated sprint speed endurance, vertical jump, and aerobic capacity in young elite male soccer players. J Strength Cond Res. 2017;25(9):2364-2370.

14. Stolen T, Chamari K, Castagna C, et al. Physiology of soccer: an update. Sports Med. 2005;35(6):501-536.

15. Reis VM, Garrido ND, Vianna J, et al. Energy cost of isolated resistance exercises across low- to high-intensities. PLoS One. 2017;12.

16. Scott CB, Reis VM. Steady state models provide an invalid estimate of intermittent resistance-exercise energy costs. Eur J Human Movement. 2014;33:70-78.

17. Paul DJ, Nassis GP. Testing strength and power in soccer players: the application of conventional and traditional methods of assessment. $J$ Strength Cond Res. 2015;29(6):1748-1758.

18. Śliwowski R, Grygorowicz M, Hojszyk R, et al. The isokinetic strength profile of elite soccer players according to playing position. PLoS One. $2017 ; 12$.

19. Misook H, Dongwook H. The relationship between knee joint angle and knee flexor and extensor muscle strength. J Phys Ther. Sci. 2017;29(4):662-664.

20. Ribeiro F, Lépine PA, Garceau BC, et al. Test-retest reliability of lower limb isokinetic endurance in COPD: A comparison of angular velocities. Int J Chron Obstruct Pulmon Dis. 2015;18:1163-1172.

21. Bosquet L, Gouadec K, Berryman N, et al. The total work measured during a high intensity isokinetic fatigue test is associated with anaerobic work capacity. J Sports Sci Med. 2016;15(1):126-130. 